\title{
THE "STRING SYNDROME" SEEN AS A COMPLICATION OF ARRUGA'S CERCLAGE SUTURE
}

\author{
BY \\ NEIL MANSON \\ Department of Ophthalmology, Royal Victoria Infirmary, Newcastle upon Tyne
}

OF the techniques used in the surgery of retinal detachment to appose choroid and retina, Arruga's equatorial cerclage suture (see Arruga, 1958a, b, 1961, 1962), which is a modification of a technique used by Schepens, Okamura, and Brockhurst (1957, 1958), is probably one of the simplest yet most rewarding.

The equatorial cerclage suture seals the intra-retinal space at the equator, at first mechanically and subsequently by a sterile choroiditis, allowing the retina of the posterior part of the globe within the suture to remain flat. Anterior to this apposition of choroid and retina there may remain subretinal fluid, tears, and other pathology, but theoretically, as long as the seal is complete, the retina of the posterior pole will remain flat. The technique has been used with increasing frequency as a primary procedure in the Ophthalmic Department of the Royal Victoria Infirmary, Newcastle upon Tyne. Its useful application is enhanced by the ability to combine it with other techniques.

Our experience has inevitably included failures, some of which show several characteristic features which, for the lack of a suitable collective term, we call the "string syndrome". The fundus appearance of a cerclage suture is shown in Fig. 1 (opposite).

Of the 36 cases treated with a cerclage suture, four have exhibited the "string syndrome" post-operatively. The constancy of the clinical signs associated with what appears to be inevitable amaurosis would appear to warrant its consideration and discussion in some detail.

The signs of this syndrome are oedema of the lids, true proptosis of the globe, chemosis of the conjunctiva, uveitis, and ocular hypotension. The onset of these signs is variable, having been seen between the 4th and 19th post-operative day. The conjunctival chemosis is gross and in one case the conjunctiva prolapsed through the palpebral aperture and had ultimately to be excised (Fig. 2).

Arruga described, among the complications of his cerclage suture the occurrence of a rather marked conjunctival reaction. We have also experienced this, but the nature of this reaction and the appearance of the conjunctiva is unmistakably different from the type of conjunctival reaction seen in the string syndrome. The former is a much milder form of reaction (Fig. 3) and invariably resolves quite quickly. The most striking difference between the two reactions is the colour of the conjunctiva. In the case which resolves quickly it has a rosy hue, although it may be quite chemotic, in the serious string type it has more the colour of a ripe tomato.

In the string syndrome, while the cornea remains clear, the anterior chamber is very deep, the iris being fixed and assuming a distinct green colour (Fig. 4).

* Received for publication April 26, 1963. 
(1)
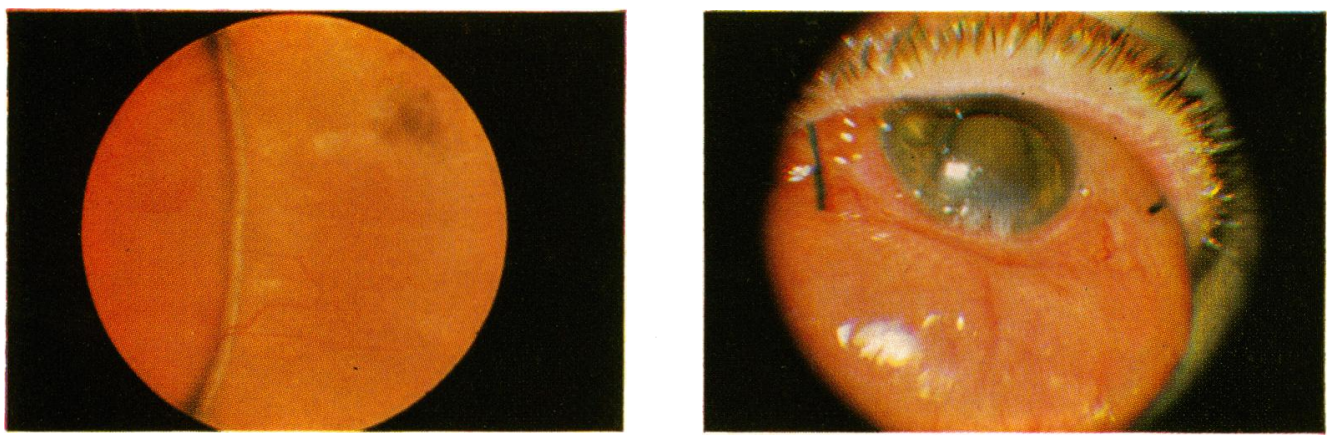

(2)

(3)

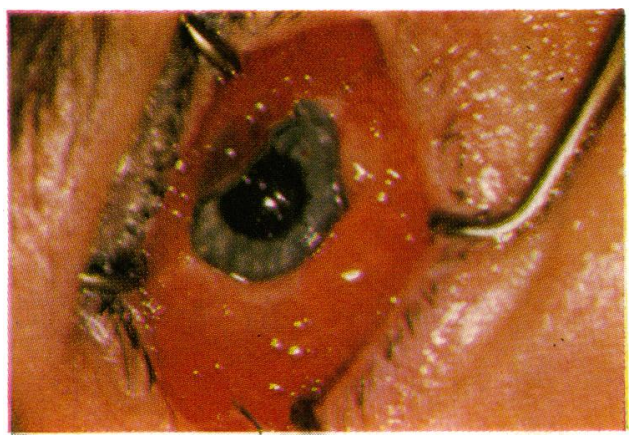

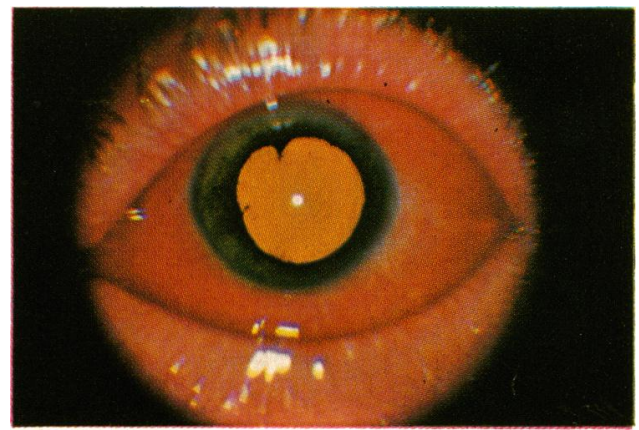

(4)

Fig. 1.-Fundus appearance in the line of the cerclage suture, showing pigmentation.

Fig. 2.-Severe conjunctival reaction prolapsing through palpebral aperture.

FIG. 3.-Mild form of conjunctival reaction.

Fig. 4.-String syndrome showing posterior synechiae. 
An aqueous flare is present which responds, if at all, only slowly to treatment with steroids. This syndrome persists for many weeks, and although it ultimately resolves, secondary changes persist, and it appears that, whatever the immediate post-operative state of the retina, the end-result is a complete detachment. This ocular reaction is associated with a great deal of pain, but there has been no evidence of infection, bacteriological or clinical, except in one case in which there was a prolonged prolapse of conjunctiva (Fig. 2); in this case secondary infection with a hospital Staphylococcus aureus occurred after many weeks and resolved only on treatment with Celbenin.

In each case examined, the intra-ocular pressure has been persistently between 4 and $6 \mathrm{~mm}$. $\mathrm{Hg}$ (Schiötz weighted tonometer), this being indicative of absent ciliary function. Resumption of ciliary function has not occurred during a limited followup period, the longest having been 10 months.

It is interesting to note that Bárány (1955) commented on similar findings in rabbits when a constricting cuff was applied behind the equator of the eye. He used a balloon, so placed around the dislocated globe that the neck of the balloon constricted the globe behind the equator (Fig. 5). A few hours after such a procedure, the tension was raised and the conjunctiva was slightly oedematous and without haemorrhages. The pupil remained normally active and there was little or no aqueous flare. There was engorgement of the pericorneal vessels. 18 to 24 hours after constriction, haemorrhages occurred in the oedematous conjunctiva, the pupil was constricted, and an aqueous flare developed, the intra-ocular pressure being raised.

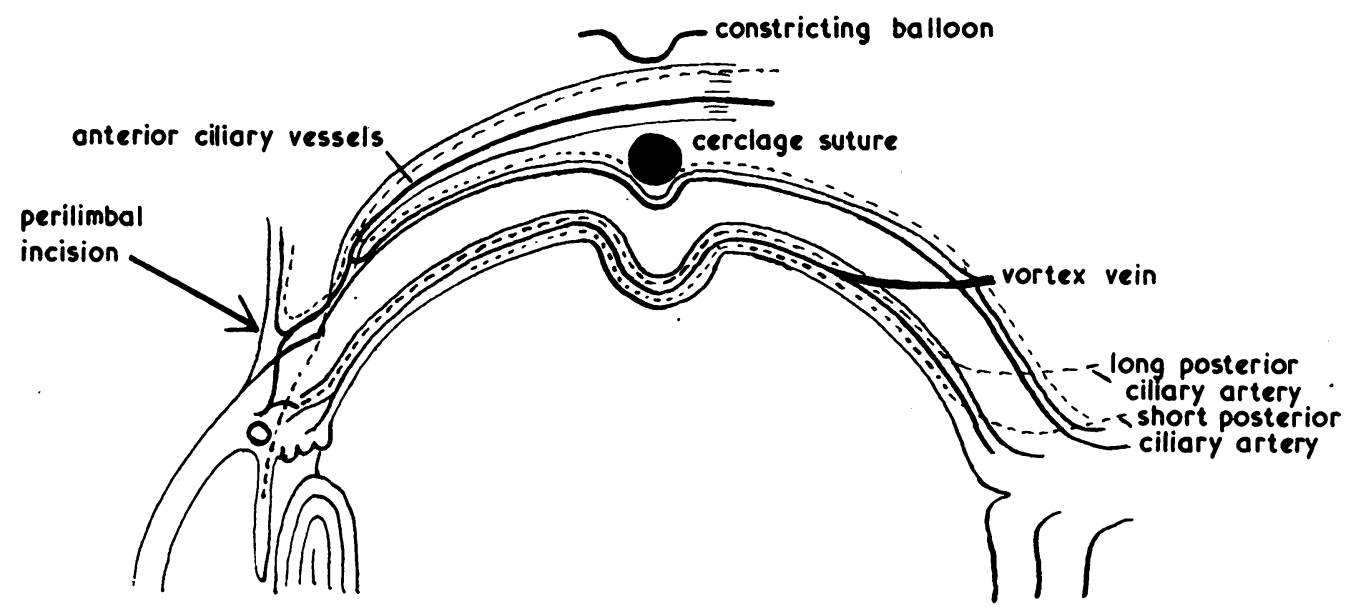

FIG. 5.-Cerclage suture and constricting balloon shown diagrammatically in section.

The similarity between this condition produced experimentally in rabbits and the string syndrome is striking in most respects. An initial rise in intra-ocular pressure which was not maintained was found in only one human case, but by the very nature of the cases and the wish not to interfere with post-operative detachments too frequently, comprehensive tonometric curves were not kept. Frank conjunctival haemorrhages were not seen in human cases. In the discussion of this condition it will be pointed out that the constricting balloon and the cerclage suture are probably 
in different positions in relation to the extra-ocular muscles. Intra-ocular hypertension is not unknown as a complication of retinal surgery, and has been accounted for by the pushing forwards of the ciliary body with the occlusion of the drainage angle (Ross, 1960). This may have occurred in the rabbits. To ascertain the state of the angle in humans exhibiting this syndrome gonioscopy was performed, and in each case the angle was found to be open with no neo-vascularization or goniosynechiae.

\section{Case Report}

A man aged 58 years, a high myope, had an old complete retinal detachment in the right eye and the visual acuity was less than 6/60 in the left eye, which showed incipient lens opacities and a temporal detachment.

Operation.-An Arruga's cerclage suture was placed $14.5 \mathrm{~mm}$. from the limbus in association with diathermy puncture and limited surface diathermy.

7th Post-operative Day: A red reflex only was visible.

13th Post-operative Day: The digital ocular tension was raised, the eye being irritable with a shallow anterior chamber (Fig. 6i).

19th Post-operative Day: The conjunctiva was chemotic and dark red in colour. The iris was green with posterior synechiae and a temporal retinal detachment was seen to be still present.

23rd Post-operative Day: The anterior chamber was now deep, the iris remained green (Fig. 4) but was markedly stepped suggesting complete posterior synechiae (Fig. 6ii).

25th Post-operative Day: The anterior chamber remained deep and there was a choroidal detachment.

34th Post-operative Day: The ocular tension was very low. The pupil was irregular and the anterior chamber was assuming a more normal depth (Fig. 6iii). The lenticular opacities were increasing.

7 Months Post-operatively: There was chronic ciliary injection and the intra-ocular pressure was $4 \mathrm{~mm}$. Hg. Gonioscopy showed a normal open angle and the retina was completely detached. Exophthalmometer readings showed right $15 \mathrm{~mm}$. and left $20 \mathrm{~mm}$.

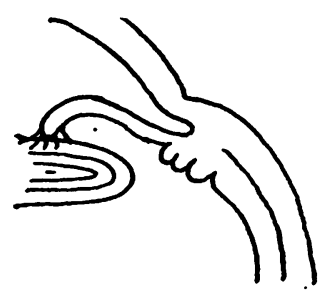

(i)

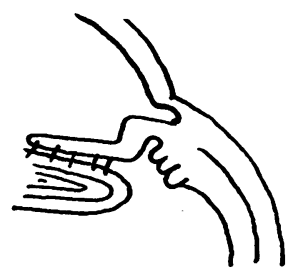

(ii)

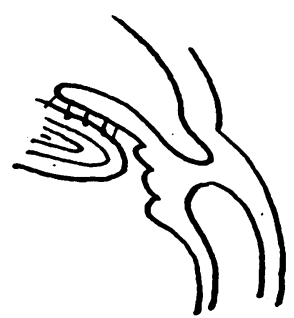

(iii)

FIG. 6.-(i) Posterior synechiae with iris bombé.

(ii) Complete posterior synechiae causing stepped iris and deep anterior chamber.

(iii) Choroidal and ciliary body detachment allowing anterior chamber to assume a more normal depth.

\section{Causes of the String Syndrome}

Duke-Elder (1961) comments on the dangers of drawing anatomical deductions from inadequate clinical material. It may however, be more permissible to find an explanation for a clinical sign in anatomical facts.

Venous Drainage.-The vortex veins drain the whole of the choroid, ciliary body, and iris via the chorio-capillaris. The venous blood from the iris, ciliary body, and choroid is channelled via the chorio-capillaris and choroidal venules into larger veins (vortex veins) which commence just behind the equatorial region. As with veins 
elsewhere in the body, there is variation in the system and malposition may occur, but normally the nasal veins are placed more anterior than the temporal veins. From the anterior and outer part of the ciliary muscles, an anterior ciliary venous system drains to the episcleral plexus by piercing the sclera posterior to the limbus and then drains into the aqueous veins. These anterior ciliary veins receive tributaries from Schlemm's canal, so contributing, with the aqueous veins, to the aqueous drainage.

It is therefore possible that the signs of the string syndrome could be due to occlusion of the venous drainage of the iris, ciliary body, and anterior part of the choroid. A further danger shown by consideration of the venous drainage is that, in providing a good exposure by peritotomy and posterior stripping of conjunctiva, one may unwittingly interfere with these veins as shown in Fig. 5.

The exposure by peritotomy, whilst being the most convenient, because of its inferred interference with aqueous drainage, has been replaced by the originally described incisions through the conjunctiva in each quadrant.

Arterial Supply.-The supply of the short posterior ciliary arteries is likely to be interfered with $(a)$ in the episcleral arterial plexus, and $(b)$ where some of these vessels travel in the outer part of the choroid to the equatorial region before terminating as choroidal capillaries.

The long posterior ciliary arteries, running forwards as medial and lateral vessels in the supra-choroidal space to join the anterior ciliary arteries in the formation of the major arterial circle of the iris, may be directly occluded by the cerclage suture.

The anterior ciliary arteries arising from the muscular vessels are important in this regard. Large perforating vessels pass into the anterior part of the ciliary body to help form the major circle. Resection of the muscles with subsequent fibrosis may interfere with this supply. Such devascularization is the aim of cyclo-anaemization operations, which lower the intra-ocular pressure by interfering with the ciliary blood supply. It is for a similar reason that the removal of more than two extraocular muscles at any one operation is contraindicated.

The cerclage suture, as performed in man, avoids direct constriction of these vessels, the suture being placed between the globe and the extra-ocular muscles. In Bárány's experimental rabbits, however, these vessels would be included in the constricting cuff of the balloon (Fig. 5).

Electro-coagulation by surface diathermy anterior to the equator may affect all the vessels mentioned, particularly when large areas are treated or an encircling barrage has been performed in relation to the suture.

Consideration of the lymphatic drainage is unnecessary as it is non-existent.

Amongst the signs of the string syndrome for which there appears to be no anatomical basis, are the oedema of the lids and the proptosis, unless the latter is due to generalized vascular engorgement and stasis with more permanent proptosis resulting from orbital fibrosis.

Complications of the Cerclage Suture.- It has been said that the type of mild conjunctival reaction shown (Fig. 3) is due to the string and that any other condition is due to excessive electro-coagulation. There is, however, no anatomical support for this 
comment, the conjunctival blood supply and drainage not being involved or interfered with by the cerclage suture. Further, the choroido-retinal reaction due to diathermy and that due to the application of a cerclage suture to the globe are the same. Forced apposition of the histological layers of the globe and interference with the choriocapillaris probably produce pigmentary and glial proliferation. The clinical appearance of pigmentation in the line of the cerclage suture (Fig. 1) implies a similar reaction to that obtained with surface electrocoagulation. It would therefore be difficult to explain the signs by one factor in preference to another or by a combination of factors.

In one case (see Case Report), the position of the iris was of great interest and implies that many of the signs of the string syndrome could be due to a cyclitis, posterior synechiae producing an iris bombé (Fig. 6i), and the posterior synechiae then becoming complete and retracting the iris to form a deep anterior chamber. These stages would be associated with the rise in intra-ocular pressure which was noted. Later a ciliary body detachment, the.ciliary body being attached only to the scleral spur and anterior part of the choroid, would account for the cessation of ciliary function and allow the iris to become more normally placed, so reducing the depth of the anterior chamber (Fig. $6 i, i i$, and iii).

Finally, three of the four cases of string syndrome were high myopes in whom ciliary function may have been inadequate initially, and whose long anatomical axis may be involved in some way.

\section{Summary}

Certain complications of the cerclage operation, collectively referred to as the "string syndrome", are discussed.

The thoughtful and limited application of diathermy in conjunction with the cerclage suture may avoid the occurrence of the string syndrome. It also appears that the suture itself is likely to cause vascular occlusive complications but it is possible that the configuration of some eyes may be a predisposing factor.

I should like to thank Mr. H. Vernon Ingram and Mr. L. H. Lake for the facilities they have afforded me with respect to these cases, and the Board of Governors of the United Newcastle upon Tyne Hospitals, for generous aid from their endowment fund.

\section{REFERENCES}

ArRugA, H. (1958a). Bull. Soc. franc. Ophtal., 71, 571.

(1958b). Arch. Soc. oftal. hisp.-amer., 18, 55.

(1961). Ber. dtsch. ophthal. Ges. Heidelberg, 64, 480.

(1962). Arch. Soc. oftal. hisp.-amer., 22, 813.

BÁRÁNY, E. H. (1955). In "Glaucoma: a Symposium”, ed. S. Duke-Elder, C.I.O.M.S. Blackwell, Oxford. pp. 91-104.

DUKE-ELDER, S. (1961). "System of Ophthalmology", vol. 2, p. 352. Kimpton, London.

Ross, M. G. (1960). "Importance of the Vitreous Body in Retinal Surgery", in "II Conf. Retina Foundation", ed. C. L. Schepens. Mosby, St. Louis.

SCHEPENS, C. L., OKAMURA, I. D., and BrockHURST, R. J. (1957). A.M.A. Arch. Ophthal., 58, 79. 